Meta

Journal des traducteurs

Translators' Journal

\title{
Collège européen des traducteurs littéraires de Seneffe
}

\section{Françoise Wuilmart}

Volume 44, numéro 3, septembre 1999

URI : https://id.erudit.org/iderudit/004623ar

DOI : https://doi.org/10.7202/004623ar

Aller au sommaire du numéro

Éditeur(s)

Les Presses de l'Université de Montréal

ISSN

0026-0452 (imprimé)

1492-1421 (numérique)

Découvrir la revue

Citer cette note

Wuilmart, F. (1999). Collège européen des traducteurs littéraires de Seneffe. Meta, 44(3), 522-522. https://doi.org/10.7202/004623ar d'utilisation que vous pouvez consulter en ligne.

https://apropos.erudit.org/fr/usagers/politique-dutilisation/ 


\section{NDLR}

Le centre de Straelen a servi en quelque sorte de modèle pour créer le Collège International des Traducteurs Littéraires (CITL) d'Arles, pour le français, le centre de Norwich, en Grande-Bretagne pour l'anglais, celui de Procida pour l'italien, celui de Visby pour le suédois et celui de Tarazona pour l'espagnol. On sait d'autre part qu'il existe quelques programmes universitaires de formation en traduction littéraires, par exemple à Düsseldorf (anglais et français) et à Münich (anglais) en Allemagne, à Bruxelles en Belgique.

\section{Collège européen des traducteurs littéraires de Seneffe}

(Sous l'égide de la Communauté française de Belgique, avec le soutien del'Union européenne)

Depuis une vingtaine d'années, il semble bien que I'on s'intéresse de plus en plus au sort du traducteur littéraire. Sans doute a-t-on enfin compris que la qualité de la traduction en général était étroitement liée au confort de cet incontournable ambassadeur culturel.

Une des initiatives allant dans ce sens est la création de Collèges de traducteurs littéraires, à ce jour au nombre de douze en Europe. Par définition le Collège est censé offrir au résident un environnement idéal lui permettant de mener à bien ses projets de traduction.

C'est en 1996 que le collège de Seneffe vit le jour, grâce aux aides financières conjointes du ministère de la Culture de la Communauté française de Belgique, et de la Commission européenne. Installées dans les dépendances rénovées du Château de Seneffe (à une trentaine de kilomètres de Bruxelles), au cœur d'un merveilleux parc de 24 hectares, ses bâtisses blanches, entourant un vaste patio agrémenté d'une fontaine, abritent 18 chambres très confortables, une bibliothèque riche de multiples ouvrages de références et d'un plateau informatique, une salle de télévision, une grande salle à manger et un très beau salon. La particularité du Collège de Seneffe est son infrastructure hôtelière: Le résident y est complètement pris en charge: logé, nourri, chauffé, blanchi, il a aussi à sa disposition un service de téléphone et de fax.

Le Collège de Seneffe accueille toutes les combinaisons linguistiques, mais donne toutefois la priorité aux traducteurs de littérature belge de langue française, qui, en sus de la prise en charge totale, bénéficient d'un per diem s'ils sont déjà en possession d'un contrat d'édition. C'est d'ailleurs dans cette optique que le Collège met à la disposition des résidents la quasi-totalité des œuvres d'écrivains belges francophones et invite régulièrement les auteurs contemporains à des séances de lectures et de séminaires. Ainsi, les traducteurs trouvent-ils ici l'occasion de rencontrer leurs auteurs et même de travailler avec eux. De surcroît, il n'est pas rare que le Collège réunisse à la même période les traducteurs d'un même auteur, voire d'une même œuvre. Dans ce cas, les échanges et les débats ainsi rendus possibles entre les traducteurs penchés sur un même texte, sont des plus fructueux.

Le Collège de Seneffe organise aussi réguliè rement des spectacles de théâtre à la seule intention de ses résidents, manière originale de faire découvrir aux traducteurs étrangers le théâtre belge, allant de Crommelynck à Verheggen en passant par Michaud et Lejeune. Ce qui ne gâte rien, c'est que ces pièces sont jouées par l'excellente troupe du Théatre-Poème, dans un lieu idyllique: Le Petit Théâtre baroque, situé dans le parc du château, et depuis sa restauration, devenu monument classé.

N'oublions pas non plus l'importance de la grande convivialité qui règne ici durant la session entre ces traducteurs venus de tous les coins du monde et réunis par un même amour du métier. Jusqu'ici, l'expérience du Collège de Seneffe a montré que les traducteurs littéraires, champions de la tolérance par essence, puisque toujours tournés vers l'Autre et au service de l'Autre, pouvaient cohabiter dans le plus grand bonheur et la plus grande joie.

Enfin, pour marquer sa reconnaissance envers ceux ou celles qui contribuent au rayonnement de la littérature belge à l'étranger par la qualité de leurs traductions, le Collège décerne chaque année un prix de traduction d'un montant de 200000 francs belges. En 1997, le prix est alléau Hollandais, Ernst van Altena, la deuxième année à une anglaise, Anne-M arie Glasheen et cette année, c'est au hongrois Janos Lackfi qu'ira la récompense.

Avis donc aux amateurs, qui, pour obtenir de plus amples renseignements, peuvent s'adresser à la directrice du Collège, Françoise Wuilmart. (cetl@compuserve.com) 